\title{
Review of multidrug-resistant and extensively drug-resistant TB: global perspectives with a focus on sub-Saharan Africa
}

\author{
Giovanni Battista Migliori', Keertan Dheda ${ }^{2,3}$, Rosella Centis', Peter Mwaba ${ }^{4}$, Matthew Bates ${ }^{3,4}$, Justin O’Grady ${ }^{3,4}$, \\ Michael Hoelscher ${ }^{5}$ and Alimuddin Zumla ${ }^{3,4}$ \\ 1 WHO Collaborating Centre for TB and Lung Diseases, Fondazione S. Mangeri, Tradate, Italy \\ 2 Lung Infection and Immunity Unit, University of Cape Town, Cape Town, South Africa \\ 3 Centre for Infectious Diseases and International Health, University College London Medical School, London, UK \\ 4 UNZA-UCLMS Research and Training Project and University Teaching Hospital, Lusaka, Zambia \\ 5 Department of Infectious Diseases \& Tropical Medicine, Klinikum of the Ludwig-Maximilians University of Munich, Munich, Germany
}

Summary Tuberculosis (TB) remains a global emergency and is responsible for 1.7 million deaths annually. Widespread global misuse of isoniazid and rifampicin over three decades has resulted in emergence of the ominous spread of multidrug-resistant TB (MDR-TB) and extensively drug-resistant TB (XDR-TB) globally. These difficult to treat resistant forms of TB are increasingly seen in Asia, Eastern Europe, South America and sub-Saharan Africa, disrupting TB and HIV control programmes. We review the latest available global epidemiological and clinical evidence on drug-resistant TB in HIV-infected and uninfected populations, with focus on Africa where data are scanty because of poor diagnostic and reporting facilities. The difficult management and infection control problems posed by drug-resistant $\mathrm{TB}$ in HIV-infected patients are discussed. Given the increasing current global trends in MDR-TB, aggressive preventive and management strategies are urgently required to avoid disruption of global TB control efforts. The data suggest that existing interventions, public health systems and TB and HIV programmes must be strengthened significantly. Political and funder commitment is essential to curb the spread of drug-resistant TB.

keywords tuberculosis, drugs, MDR-TB, XDR-TB, epidemiology, microbiology, treatment

\section{Introduction}

Efforts to control the global tuberculosis (TB) epidemic have now been complicated by the emergence of strains of Mycobacterium tuberculosis, which are resistant to one or more anti-TB drugs. Multidrug-resistance originates from misuse of anti-TB drugs by physicians, patients and producers (Matteelli et al. 2007; Migliori et al. 2009) (Figure 1). The identification and spread of multidrugresistant TB (MDR-TB) (Pablos-Méndez et al. 1998; Espinal et al. 2001; Shah et al. 2005; WHO and IUATLD, 2008; Wright et al. 2009), and more recently, of extensively drug-resistant TB (XDR-TB) globally (Holtz et al. 2005; Shah et al. 2005; Centers for Disease Control and Prevention, 2006; WHO, 2010) is increasingly becoming a major threat to achieving the projected goal of TB control and elimination by the year 2050 as advocated by the Millennium Development Goals (MDGs) and Stop TB
Partnership. This review discusses the clinical epidemiology, microbiology, diagnosis, treatment and prevention aspects of drug-resistant TB in HIV-infected and uninfected adults globally, with special focus on MDR-/XDR-TB in resource-limited settings, with an emphasis on sub-Saharan Africa because emerging clinical experience data shows that drug-resistant TB is now more widespread than previously thought.

\section{Methods}

A systematic review of all relevant publications on drugresistant TB was performed via a Medline search using the key words 'Tuberculosis', 'drug resistance' 'MDR-TB' and 'XDR-TB', with the words 'epidemiology, diagnosis, treatment, drug resistance genes, TB control, epidemiology, management, TB control programmes, Africa and prevention' covering the period 31st March 2000 to 1st 
M. tuberculosis strains in the population

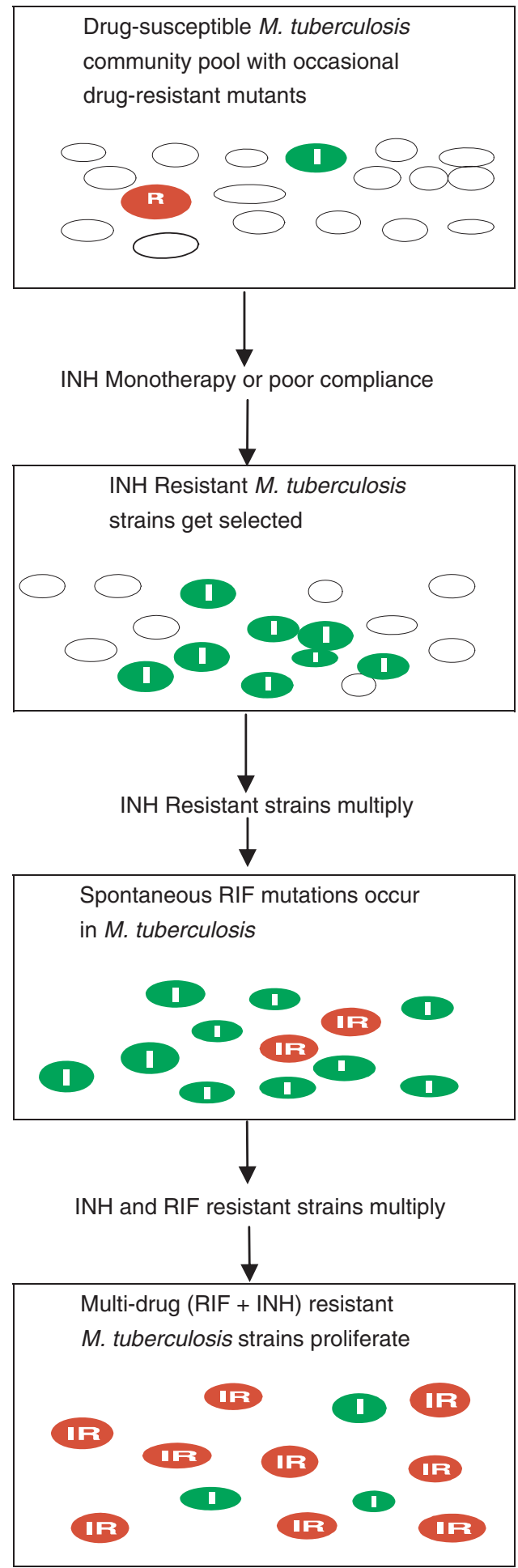

Figure I Evolution of multidrug resistance.
April 2010, and all relevant literature from English language medical journals were reviewed. In addition, the US Centers for Disease Control and Prevention (CDC) Morbidity and Mortality Weekly Report (MMWR) was searched for reports on MDR-TB and XDR-TB published from 2000 to the present and quoted in the review based on priority criteria. Further sources of information were data emerging from current studies and databases on MDR-/XDR-TB in South Africa, WHO publications on TB and drug-resistant TB published over the past 15 years (Schaaf \& Zumla 2009) and relevant chapters from two recently published textbooks on TB (Schaaf \& Zumla 2009; Zumla \& Schaaf 2009).

\section{Definitions for drug-resistant TB}

The definitions of drug-susceptible and drug-resistant TB are given in Table 1. 'Pan-susceptible TB' is defined as TB caused by Mycobacterium tuberculosis (M. tuberculosis) strains that are susceptible to all first-line anti-TB drugs. MDR-TB is defined as resistance to the two key first-line anti-TB drugs, isoniazid (INH) and rifampicin (RMP/RIF) (Pablos-Méndez et al. 1998; Espinal et al. 2001; WHO and IUATLD, 2008; Wright et al. 2009; WHO, 2010). The term XDR-TB appeared in the literature for the first time in March 2006, in a report jointly published by WHO and CDC, to describe a very aggressive form of disease characterised by high mortality rates (Centers for Disease Control and Prevention, 2006). It is presently defined as TB caused by strains of $M$. tuberculosis, which are the following: (a) resistant to at least INH and RMP (i.e. MDR-TB), (b) plus any fluoroquinolone, and (c) to at least

Table I Definitions of Drug-susceptible and drug-resistant tuberculosis (TB)

Pan (Totally)-susceptible TB

Tuberculosis caused by Mycobacterium tuberculosis strains susceptible to all first-line anti-TB drugs.

INH Monoresistant TB

Tuberculosis caused by M. tuberculosis strains resistant only to $\mathrm{INH}$

Multidrug-resistant tuberculosis (MDR-TB)

Tuberculosis caused by $M$. tuberculosis strains resistant to at least two first-line anti-TB drugs INH and rifampicin (RMP)

Extensively drug-resistant tuberculosis (XDR-TB)

Tuberculosis caused by M. tuberculosis resistant to RMP and INH plus any fluoroquinolone, and at least one of the three following injectable drugs: capreomycin, kanamycin or amikacin Pan (Totally)-resistant TB

Tuberculosis caused by M. tuberculosis resistant to all first and second-line anti-TB drugs

$\mathrm{INH}$, isoniazid. 


\section{G. B. Migliori et al. Drug-resistant TB}

one of three injectable drugs used in anti-TB treatment, capreomycin, kanamycin or amikacin (Holtz \& Cegielski 2007; Migliori et al. 2007).

\section{Epidemiology of MDR-TB and XDR-TB}

The latest data on the extent of drug-resistant TB are summarised in the 2010 WHO MDR-/XDR-TB Global report on surveillance and response (WHO, 2010). The proportions of MDR-TB among all new TB cases is shown in Figure 2. MDR-TB caused an estimated 150000 deaths in 2008. The $2010 \mathrm{WHO}$ report estimates that 440000 MDR-TB cases occurred in 2008 (3.6\% of the estimated total incident TB episodes). Of these, 360000 were among new TB cases, and 94000 were among individuals previously treated. Data on drug-resistant TB from Africa are very scarce. India and China constitute approximately 50\% of the global burden of MDR-TB, followed by the Russian Federation (9\%). Eastern Europe and Central Asia have reported very high proportions of MDR-TB among new cases (Balabanova et al. 2005; Migliori et al. 2010). The Russian Federation's surveillance data from 12 of its oblasts and republics reported proportions of $23.8-28.3 \%$ MDR$\mathrm{TB}$ among new TB cases in three of its oblasts in the northwestern part of the country.

Role of HIV in fuelling the drug-resistant TB epidemic

About $8 \%$ of all infections with $M$. tuberculosis occur in people with HIV, making it the most important opportunistic infection worldwide. Over the past two decades, the HIV pandemic has been the most important factor for increased numbers of TB cases being reported in subSaharan Africa (Elliott et al. 1993; Habeenzu et al. 2007; Kehinde et al. 2007; Umubyeyi et al. 2007; Meskel et al. 2008). The spread of the TB epidemic is closely related to the high HIV infection rates in the general population. Recently, co-infection of the two pathogens, M. tuberculosis and HIV, has become more noticeable in Eastern Europe and in Asia (Kingkaew et al. 2009). Sub-Saharan Africa bears the burden of both very high TB incidence and the highest human immunodeficiency virus (HIV) prevalence rates in the world, and now represents $14 \%$ of the global burden of new MDR-TB cases (Amor et al. 2008; WHO, 2010).

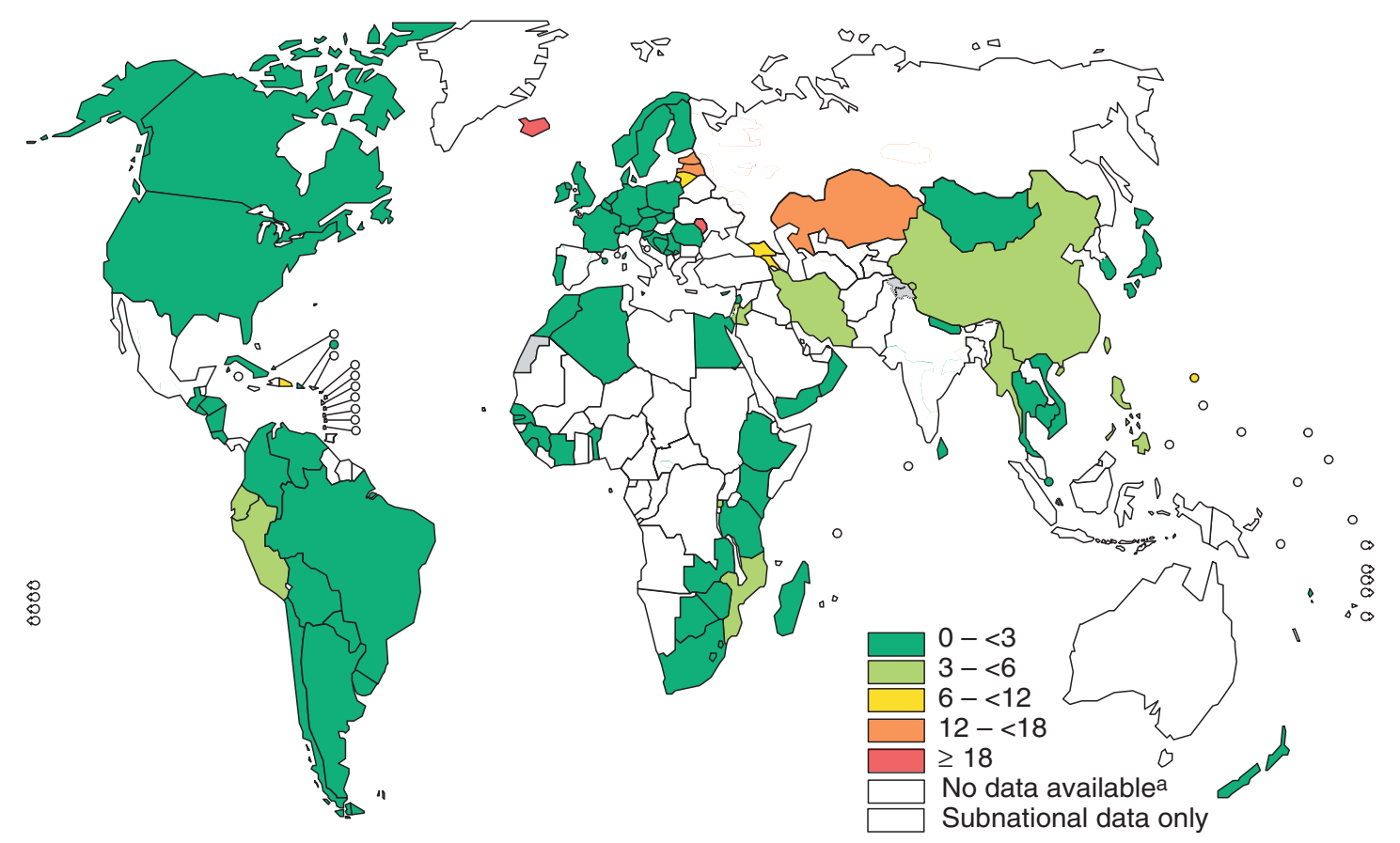

Australia, Democratic Republic of the Congo, Fiji, Guam, New Caledonia, Solomon Islands and Qatar reported data on combined new and previously treated cases.

Figure 2 Global proportions of MDR-TB among new TB cases. [Reproduced with permission from WHO, (2010)]. MDR-TB, multidrug-resistant tuberculosis. 
G. B. Migliori et al. Drug-resistant TB

\section{MDR-TB and XDR-TB in sub-Saharan Africa}

There are scanty data about MDR-TB trends in Africa. According to the 2010 Global WHO Report (WHO, 2010), only 22 of 46 countries of the African Region have provided data on drug-resistant TB (12 having conducted a nationwide survey since 2000 and 10 a sub-national level survey). South Africa is the only country that collects routine surveillance data. Thirty-four countries have reported MDR-TB cases and eight XDR-TB cases. Only three countries (Rwanda, the United Republic of Tanzania and South Africa) have information on the proportion of XDR-TB among MDR-TB cases. The reason why the proportion of MDR-TB among patients with TB is generally low in the African region (with a frequency ranging from $0.5 \%$ to $3.9 \%$ among new TB cases and $0.0 \%$ to $16.7 \%$ among previously treated ones) is probably related to poor laboratory facilities for drug susceptibility testing (DST), poor surveillance mechanisms and reporting procedures, outdated databases and sub-optimal coverage of the infrequent surveys. Given the high incidence of TB per population, the rates of MDR-TB cases arising per 100000 population in some southern African countries are predicted to be 5-6 times higher than those reported in China and India. The latest WHO estimate of the number of MDR-TB cases emerging in 2008 in Africa, most likely an underestimate, is 69000 cases (WHO, 2010).

Nine African countries rate among the 27 high-burden MDR-TB countries with South Africa estimated to have approximately $20000 \mathrm{MDR}-\mathrm{TB}$ cases annually. Amor and co-investigators based on data from 39/46 countries in Africa estimate that MDR-TB is likely to be more prevalent than previously recognised (Amor et al. 2008). Indeed, more recent data indicate higher rates of MDR-TB in several prevalence surveys. Recent surveys from Ethiopia (retreatment cases) (Meskel et al. 2008), Nigeria (tertiary hospital) (Kehinde et al. 2007), Zambia (prison) (Habeenzu et al. 2007) and Rwanda (retreatment cases) (Umubyeyi et al. 2007) indicate MDR-TB prevalence rates of $26 \%, 54 \%, 9.5 \%$ and $9.4 \%$, respectively. Alarming data from Botswana suggest that notification rates of all cases of TB and also of MDR-TB are increasing (Chirenda et al. 2009). If this reflects the situation in other high HIVprevalence countries where TB control programmes have been destabilised, then the situation might be worse than previously thought (Habeenzu et al. 2007; Kehinde et al. 2007; Umubyeyi et al. 2007; Amor et al. 2008; Meskel et al. 2008; Chirenda et al. 2009; Kingkaew et al. 2009).

The lack of information on drug resistance in Africa will impede planning of surveillance and activities of the national TB and HIV programmes. Current estimates of MDR-TB cases in many African countries are thus based on mathematical modelling rather than actual studies. Laboratory surveillance for MDR-TB and XDR-TB should be strengthened and expanded across the region, particularly where studies have never been carried out or data are now older than 5 years. Three groups have recently published their studies on the outcome of XDR-TB treatment from South Africa (Gandhi et al. 2006; O'Donnell et al. 2009; Wright et al. 2009; Dheda et al. 2010; Gandhi et al. 2010a,b). These studies, which are limited by their restricted study design and not representative of the general population, reveal that the drug resistance problem in South Africa is more widespread than previously thought and needs proper definition. It is evident that urgent initiation and implementation of regular comprehensive and methodologically sound surveillance studies from African countries are now needed to gauge the true extent of the TB drug-resistant problem.

\section{Microbiology of MDR-TB and XDR-TB}

Drug-resistant TB is a microbiological diagnosis based on susceptibility patterns of the M. tuberculosis strains isolated from any patient clinical sample (Table 1). Multidrug-resistant $M$. tuberculosis strains are defined as those that are resistant to INH and RMP. The frequency of resistance to multiple drugs varies geographically, and acquired (secondary) resistance is more common than primary resistance. Drug resistance in M. tuberculosis is because of the acquisition of mutations in chromosomally encoded genes (Johnson et al. 2009).

The generation of multidrug-resistance in M. tuberculosis is a consequence of accumulation of mutations primarily because of inadequate or intermittent therapy or noncompliance (Figure 1). Resistance to INH is because of mutations in the kat $G$ gene, and about half of all isolates with kat $G$ mutations have an amino acid replacement at codon 315. The $r p o B$ gene, which encodes the $\beta$-subunit of RNA polymerase, harbours a mutation in an 81 -bp region in about $95 \%$ of RMP-resistant M. tuberculosis strains recovered globally. Mutations in the $p n c A$ gene can cause pyrazinamide (PZA) resistance in approximately $70 \%$ of clinical isolates. Approximately $65 \%$ of clinical isolates resistant to ethambutol (EMB) have a mutation in the $e m b B$ gene. Clinical and genotypic characteristics of XDR-TB and MDR-TB are now being studied. The following drug resistance genes have been identified in second-line agents: gyrA in flouroquinolones, rrs for aminoglycosides Kanamycin and Amikacin, inhS, othA and ethR for ethionamide, $a l r$ and $d d l$ for D-cycloserine and rrs for Capreomycin. The genotypic characteristics of M. tuberculosis isolates from non-HIV-infected patients having MDR-TB and XDR-TB evaluated by spoligotyping and the 24-locus mycobacterial 
interspersed repetitive units-variable number of tandem repeats scheme showed that the Beijing genotype was the most common genotype. The clinical features of patients infected with the Beijing genotype and the drug resistance profile of the Beijing genotype isolates were similar to those for the non-Beijing family genotype.

The genotypic analysis of MDR-TB isolates shows that commonly found mutations in drug-resistant isolates are seen from different regions of the world. Transmission chains can be defined by isolates having identical IS6110 DNA fingerprints, spoligotypes and mutations conferring resistance. Molecular studies on mycobacterial strains can give clues to nosocomial transmission of drug-resistant M. tuberculosis strains. A study at King George V (KGV) Hospital, in KwaZulu-Natal (KZN) (Pillay \& Sturm 2010) determined the role of nosocomial transmission of drugresistant TB in 26 patients infected with a new strain of M. tuberculosis during treatment. DNA fingerprints 14 of 26 patients with differing isolates matched those of other patients. Four acquired a F15/LAM4/KZN genotype, two acquired fully susceptible Beijing strains. Three of the four F15/LAM4/KZN strains were multidrug-resistant with identical fingerprint patterns, while the fourth was fully susceptible. One of these was acquired during hospitalisation and three after discharge. Both HIV-infected and noninfected patients are at risk of infection with the F15/LAM4/KZN strain in health care facilities and within the community. There is a need for new diagnostic tests (Wallis et al. 2010), which can rapidly identify drugresistant strains of $M$. tuberculosis from clinical samples so patients can be appropriately isolated to prevent nosocomial transmission.

\section{Clinical features of MDR-TB and XDR-TB}

\section{Clinical presentation, symptoms and signs}

The clinical presentation, symptoms and signs of patients with drug-resistant tuberculosis in the majority of cases do not substantially differ from that of patients with TB because of pan-susceptible M. tuberculosis strains (Gandhi et al. 2006; O’Donnell et al. 2009; Schaaf \& Dheda 2009). Co-infection with HIV further complicates the clinical picture because of other concomitant opportunistic infections (Dubrovina et al. 2008; Schaaf \& Dheda 2009; Gandhi et al. 2010; Grobusch 2010). In the early stages of $\mathrm{HIV}$ infection, the clinical features of TB are similar to those of HIV-uninfected patients. Clinical presentation becomes more atypical with progressive immunosuppression. Drug-resistant TB should be suspected in patients who fail to respond to the intensive phase of standard short-course anti-TB therapy, or those who have: had TB previously; a history of poor compliance and substance abuse; been in contact with patients with drug-resistant TB. At least half of the patients with drug-resistant TB have none of these risk factors thus clinical awareness and a high degree of suspicion are required.

\section{Establishing a diagnosis of drug-resistant TB}

Accurate and rapid diagnosis of drug-resistant TB is of paramount importance for instituting appropriate clinical management and appropriate infection control measures (Schaaf \& Dheda 2009; Wallis et al. 2010). The diagnosis is usually suspected on clinical and radiological grounds, and all health practitioners in high TB and HIV endemic areas must have a high index of suspicion of drug-resistant TB. The diagnosis of drug-resistant TB can only be confirmed by molecular microbiological studies as described earlier. When pulmonary TB is suspected clinically in high TB and HIV/AIDS endemic areas, other pulmonary infections should be excluded. These include Pneumococcus spp., Chlamydiae pneumoniae, Mycoplasma spp., Legionelle spp., non-tuberculous mycobacteria (e.g. Mycobacterium avium complex), Cryptococcus spp., Histoplasma spp., cytomegalovirus, Aspergillus spp., Candida spp., and Pneumocystis jeroveci. Other noninfectious complications also need to be considered such as sarcoidosis, Kaposi's sarcoma, lymphoproliferative diseases and throe malignancies. The diagnostic steps in the management of HIV-infected and HIV-uninfected patients with drug-resistant $\mathrm{TB}$ are similar to those of drug sensitive TB using conventional diagnostic algorithms. All M. tuberculosis isolates should be subject to DST (Moore et al. 2006; Pai et al. 2006; Hoek et al. 2008; Martin et al. 2008; Pietzka et al. 2009). This usually involves direct or indirect DST in selective media using liquid or solid culture, and this may take several weeks before results are available. Furthermore, the costs of these tests are prohibitive, and laboratory capacity and TB programme budgets in African countries are severely limited. Recent advances in molecular technology for DST have improved our ability to rapidly diagnose MDR-TB and XDR-TB (Pai et al. 2006; Wallis et al. 2010). Appropriate budgetary investments into expansion of laboratory capacity to rapidly diagnose drug-resistant $\mathrm{TB}$ and instituting treatment quickly are now required.

\section{Drug treatment of drug-resistant TB}

When a diagnosis of MDR-TB is made, it gives the attending physician another chance to cure the patient, thus it is imperative that the best and most comprehensive possible treatment regimen be used to successfully treat the 
G. B. Migliori et al. Drug-resistant TB

patient. Failure to effectively treat the patient will lead to further amplification of drug resistance leading to the development of XDR-TB (Espinal et al. 2000; Espinal 2003; Quy et al. 2003; Chan et al. 2004; Saravia et al. 2005; Gandhi et al. 2010; Grobusch 2010). Patients with MDR TB on treatment should be nursed in isolation until sputum smear microscopy and cultures turn negative. Close monitoring of the patient with DOTS and follow-up is required after patient is discharged from the treatment facility. Table 2 shows drug groups and treatment regimens that are recommended for MDR and XDR in adults. Table 3 shows the serious adverse events associated with these drugs. Available first-line drugs comprise RMP, INH,
EMB, PZA and streptomycin (SM). INH and RMP are the most potent of these drugs. Second-line drugs are amikacin, capreomycin, cycloserine, linezolid, prothionamide, rifabutin and any of the flouroquinolones, e.g. moxifloxacin, levofloxacin, ofloxacin, gatifloxacin. The treatment of patients with MDR-TB and XDR-TB relies on drugs that are less potent, need to be administered for a much longer time and are substantially more toxic than those used to treat TB caused by drug-susceptible strains. The cost of a second-line drug regimen is much higher: up to thousands of dollars compared to the cost of about US\$ 20 per patient for the standard 6-month short-course, first-line chemotherapy regimen (WHO Category 1 ). The optimal duration

Table 2 Drug groups for multidrug-resistant (MDR) and extensively drug-resistant (XDR-TB) tuberculosis treatment regimens for adults [adapted from Schaaf and Dheda (2009)]

\begin{tabular}{|c|c|c|c|c|c|}
\hline \multirow[b]{2}{*}{ Drug group } & \multirow[b]{2}{*}{ Drug name } & \multicolumn{4}{|c|}{ Daily dosage in $\mathrm{mg}$ for adults $(<33 \mathrm{~kg}-$ dose in $\mathrm{mg} / \mathrm{kg})$} \\
\hline & & $\begin{array}{l}<33 \mathrm{~kg} \text { dose } \\
\text { in } \mathrm{mg} / \mathrm{kg}\end{array}$ & $33-50 \mathrm{~kg}$ & $51-70 \mathrm{~kg}$ & $\begin{array}{l}>70 \mathrm{~kg}(\text { also } \\
\text { maximum } \\
\text { dose) }\end{array}$ \\
\hline \multirow{2}{*}{$\begin{array}{l}\text { * Group 1: Oral first- } \\
\text { line drugs to which } \\
\text { the organism shows in } \\
\text { vitro susceptibility by DST }\end{array}$} & Ethambutol & 25 & $800-1200$ & $1200-1600$ & $1600-2000$ \\
\hline & Pyrazinamide & $30-40$ & $1000-1750$ & $1750-2000$ & $2000-2500$ \\
\hline \multirow{3}{*}{$\begin{array}{l}\text { †Group 2: Second- } \\
\text { line injectable agents } \\
\text { (streptomycin is first- } \\
\text { line drug - not for use } \\
\text { in MDR/XDR-TB) }\end{array}$} & Amikacin & $15-20$ & $500-750$ & $750-1000$ & 1000 \\
\hline & Kanamycin & $15-20$ & $500-750$ & $750-1000$ & 1000 \\
\hline & Capreomycin & $15-20$ & $500-750$ & $750-1000$ & 1000 \\
\hline \multirow[t]{3}{*}{$\dagger$ Group 3: Fluoroquinolones } & Ofloxacin & $15-20$ & 800 & 800 & $800-1000$ \\
\hline & Levofloxacin & $7.5-10$ & 750 & 750 & $750-1000$ \\
\hline & Moxifloxacin & $7.5-10$ & 400 & 400 & 400 \\
\hline \multirow{5}{*}{$\begin{array}{l}\text { \$Group 4: Second-line oral } \\
\text { bacteriostatic agents }\end{array}$} & Ethionamide (or prothionamide) & $15-20$ & 500 & 750 & $750-1000$ \\
\hline & & $15-20$ & 500 & 750 & $750-1000$ \\
\hline & Cycloserine (or terizidone) & $15-20$ & 500 & 750 & $750-1000$ \\
\hline & & $10-20$ & 600 & 600 & 900 \\
\hline & Para-aminosalisylic acid (PAS) & 150 & $8 \mathrm{~g}$ & $8 \mathrm{~g}$ & $8-12 \mathrm{~g}$ \\
\hline \multirow{6}{*}{$\begin{array}{l}\text { \$Group 5: Drugs of } \\
\text { unclear use in drug- } \\
\text { resistant TB treatment }\end{array}$} & High-dose isoniazid & $16-20$ & & & \\
\hline & Linezolid & \multicolumn{4}{|c|}{$\begin{array}{l}600 \mathrm{mg} \text { twice daily, but recent data suggest } 300 \\
\text { mg twice daily or } 600 \mathrm{mg} \text { or } 300 \mathrm{mg} \text { may be } \\
\text { effective (see text - New drugs) }\end{array}$} \\
\hline & Amoxicillin/clavulanate & \multicolumn{4}{|c|}{$\begin{array}{l}\text { Dosage for DR-TB not well defined. Normal } \\
\text { adult dose } 875 / 125 \mathrm{mg} \text { twice daily or } \\
500 / 125 \mathrm{mg} \text { three times daily. Higher dose } \\
\text { limited by adverse effects }\end{array}$} \\
\hline & Clarithromycin & \multicolumn{4}{|c|}{$500 \mathrm{mg}$ twice daily } \\
\hline & Thioacetazone & \multicolumn{4}{|c|}{$150 \mathrm{mg}$ daily (Contraindicated in HIV-infected patients) } \\
\hline & Imipenem/cilastatin & \multicolumn{4}{|c|}{ Usual adult dose 500-1000 mg IV 6-hourly } \\
\hline
\end{tabular}

DST, drug susceptibility testing.

*Cannot rely on DST - use as additional drug if DST result susceptible or not performed.

$\dagger$ Choose one drug in each of these groups; amikacin preferred to kanamycin in children.

tChoose one or more of these drugs to make up total of four new drugs.

SConsider use of these drugs if insufficient drugs to build an acceptable regimen with previous groups. Linezolid dosage for TB is still uncertain. Thioacetazone should not be used in HIV-infected patients. 


\section{G. B. Migliori et al. Drug-resistant TB}

\begin{tabular}{|c|c|c|}
\hline Second-line drug & Adverse clinical effect* & Tests to monitor routinely \\
\hline $\begin{array}{l}\text { Amikacin } \\
\text { Kanamycin }\end{array}$ & $\begin{array}{l}\text { Ototoxicity (cumulative } \\
\text { dose important) }\end{array}$ & $\begin{array}{l}\text { Audiology (hearing test) - } \\
\text { monthly if possible }\end{array}$ \\
\hline Capreomycin & Nephrotoxicity & $\begin{array}{l}\text { Serum creatinine and } \\
\text { potassium levels - monthly, } \\
\text { high-risk patients more often }\end{array}$ \\
\hline Fluoroquinolones & $\begin{array}{l}\text { Gastrointestinal disturbance } \\
\text { Insomnia } \\
\text { Arthralgia }\end{array}$ & $\begin{array}{l}\text { Clinical observation } \\
\text { Serum uric acid if used } \\
\text { with pyrazinamide }\end{array}$ \\
\hline $\begin{array}{l}\text { Ethionamide } \\
\text { (or protionamide) }\end{array}$ & $\begin{array}{l}\text { Gastrointestinal disturbance } \\
\text { Hepatotoxicity } \\
\text { Hypothyroidism }\end{array}$ & $\begin{array}{l}\text { Clinical observation. } \\
\text { Prevent by initially } \\
\text { splitting dose or increasing } \\
\text { dose (drug ramping) } \\
\text { Jaundice - serum alanine } \\
\text { transferase and bilirubin } \\
\text { Thyroid-stimulating } \\
\text { hormone levels (free T4) - } \\
\text { at least 6-monthly }\end{array}$ \\
\hline $\begin{array}{l}\text { Cycloserine } \\
\text { (or terizidone) }\end{array}$ & $\begin{array}{l}\text { Psychosis, seizures, } \\
\text { paresthesia, depression }\end{array}$ & $\begin{array}{l}\text { Clinical observation - } \\
\text { all patients to receive } \\
\text { preventive pyridoxine }\end{array}$ \\
\hline $\begin{array}{l}\text { Para-aminosalisylic } \\
\text { acid (PAS) }\end{array}$ & $\begin{array}{l}\text { Gastrointestinal disturbance } \\
\text { Hypothyroidism }\end{array}$ & $\begin{array}{l}\text { Clinical observation } \\
\text { Thyroid-stimulating } \\
\text { hormone levels (free T4) - } \\
\text { at least 6-monthly }\end{array}$ \\
\hline Linezolid & $\begin{array}{l}\text { Myelosuppression or } \\
\text { thrombocytopenia } \\
\text { Lactic acidosis } \\
\text { Peripheral neuropathy } \\
\text { Pancreatitis (Abdominal pain) } \\
\text { Optic neuritis } \\
\text { Drug interaction with MAOIs }\end{array}$ & $\begin{array}{l}\text { Full blood count } \\
\text { and platelets - weekly } \\
\text { at first then monthly } \\
\text { Serum lactate level } \\
\text { Symptoms of tingling } \\
\text { and numbness } \\
\text { Clinical and serum } \\
\text { amylase as indicated } \\
\text { Vision testing } \\
\text { Exclude use of monoamine } \\
\text { oxidase inhibitors }\end{array}$ \\
\hline
\end{tabular}

Table 3 Important adverse effects of second-line anti-tuberculosis drugs

*Adverse effects of first-line dugs and antiretroviral drugs in HIV-infected patients often have overlapping adverse effects.

of any given combination of anti-tuberculosis drugs for the treatment of MDR-TB and XDR-TB has not been defined yet (WHO, 2009). The effectiveness of third-line anti-TB drugs (amoxicillin-clavulanate, clarithromycin, clofazimine and linezolid, called Group 5 drugs in the WHO guidelines) remains undefined.

\section{Failure of therapy and re-treatment regimens}

Failure of therapy is associated with drug resistance, poor drug compliance or insufficient treatment duration (Sonnenberg et al. 2001; Korenromp et al. 2003; Migliori et al. 2002; Caminero 2008). Data from a large retrospective cohort ( $>5550$ cases) have provided evidence that standard short-course chemotherapy, based on first-line drugs, was inadequate to treat patients with MDR-TB (Espinal et al. 2000). A 6-year follow-up study from Taiwan found that patients treated with quinolone-containing second-line regimens were significantly less likely to relapse than those treated with first-line drugs (Chiang et al. 2006). The Category II re-treatment (regimen 2: INH-EMB-RMPPZA-SM)/1(INH-EMB-RMP-PZA)/5(INH-EMB-RMP) recommended by WHO is inadequate for settings with a high proportion of MDR-TB patients among those failing a Category I regimens (Sonnenberg et al. 2001; Migliori et al. 2002; Espinal 2003; Korenromp et al. 2003; 
G. B. Migliori et al. Drug-resistant TB

Key messages on multidrug-resistant (MDR-TB) and extensively drug-resistant (XDR-TB) tuberculosis

WHO estimates that 440000 MDR-TB cases occurred globally in 2008 and the spread of MDR-TB now threatens gains made in TB control

Recent data from South Africa show MDR-TB and XDR-TB are important causes of death in both HIV-infected and HIV-noninfected individuals

Drug-resistant TB should be suspected in patients who fail to respond to the intensive phase of standard short-course therapy, have had TB previously, have a history of poor compliance, are known contacts of patients with drug-resistant TB.

WHO infection control guidelines must be carefully followed to prevent community and nosocomial transmission of drug-resistant TB.

Drug-resistant TB requires serious attention from health personnel, governments, policy makers and funders to bring it under control

Quy et al. 2003; Saravia et al. 2005; Caminero 2008).

Treating MDR-TB patients using Category II regimens also runs the risk of further amplification of drug resistance (Caminero 2008). Hence, the common practice in highburden settings, in the absence of susceptibility data, to replace standard first-line treatment (regimen 1) with an extended treatment regimen containing SM (regimen 2) in patients failing first-line treatment, translates into adding a single drug to a failing regimen. To prevent the amplification of drug resistance, all re-treatment cases, and those failing a first-line regimen, should have their mycobacterial isolates screened by DST for RMP and INH resistance.

A number of basic rules for the management of these patients have been agreed upon recently (Ghandi et al. 2010b). The recommended regimen involves combination of a minimum of four drugs to which the M. tuberculosis isolate is likely to be susceptible (more than four could be necessary if four susceptible drugs are not available or if it is necessary to use some drugs belonging to group 5), the choice being based on a stepwise adoption through the five groups of anti-TB drugs categorised by order of efficacy, safety and costs (Table 2). The duration of the intensive phase, when an injectable agent is administered, should be at least 6 months ( 4 months after culture conversion). The continuation phase (without the injectable drug) should be prolonged until 18 months after culture conversion (Sotgiu et al. 2009). Recent data from South Africa suggest that XDR-TB patients with ofloxacin-resistant isolates fared better when treated with moxifloxacin, compared to XDRTB patients who did not receive moxifloxacin (Dheda et al. 2010). Although the optimal duration of treatment for MDR and XDR-TB is not known, a minimum of 18 months after the first negative culture (or 24 months for XDR-TB) is usually recommended (Schaaf $\&$ Dheda 2009).
MDR/XDR-TB patients are often hospitalised during the intensive phase of treatment or, in the case of XDR-TB, until they become culture-negative. Latest data from South Africa show that XDR-TB is not associated only with HIV-infected individuals (Dheda et al. 2010). Treating HIV-infected XDR-TB patients with appropriate anti-TB regimens and with Highly Active Antiretroviral Therapy (HAART) has lower mortality compared to untreated patients, and survival in HIV-infected patients is better than that previously reported. Because current management outcomes of XDR-TB patients in South Africa are poor, prevention of XDR-TB and early detection and management of MDR-TB and XDR-TB through programmatic and laboratory capacity strengthening remain an important priority for high TB and HIV/AIDS endemic areas.

\section{Treatment monitoring for drug-resistant TB}

Treatment monitoring is performed to evaluate early predictors of treatment outcome (sputum smear and culture conversion) and to ensure prevention and eventually timely management of adverse events of treatment. In a recent review on XDR-TB (Sotgiu et al. 2009), only three $(23 \%)$ of the 13 studies included in the review evaluated both median time to sputum smear and culture conversion, while four measured the median time to culture conversion. Longer treatment duration and delayed sputum smear conversion were reported in most studies among XDR-TB patients. Median time to sputum smear conversion ranged from 41 to 56 days in MDR-TB patients, while it ranged from 88 to 110 days in XDR-TB cases. The median time to culture conversion ranged from 58 to 99 days in MDR-TB cases; it was substantially different in XDR-TB patients, ranging from 60 to 195 days. In a recent study from South Africa, the culture conversion rate in XDR-TB patients was $<20 \%$ and of those who converted, $70 \%$ did so within 6 months of initiating treatment (Dheda et al. 2010).

\section{Risk factors and treatment outcomes of MDR-TB}

There have been several published reports on the treatment outcomes of MDR-TB treated with second-line drugs but methods used to assess outcome varied. Some focused on efficacy of treatment and reported only those who had adequate adherence to treatment and sufficient follow-up data. This approach may have overestimated the proportion of patients with a successful outcome (Goble et al. 1993; Park et al. 1998; Chan et al. 2004). Others reported outcome of the whole cohort of patients without excluding any cases from outcome analysis (Flament-Saillour et al. 1999; Kim et al. 2001). Laserson et al. (2005) proposed standardised definitions for outcome of MDR-TB 
treatment, which have enabled international comparison but the definition of treatment has not yet been standardised (Chiang et al. 2009). Nevertheless, it is clear that defaulting is one of the major challenges in the treatment of MDR-TB.

Risk factors for adverse treatment outcome in patients with MDR-TB and XDR-TB have been recently reviewed in detail (Dheda et al. 2010; Gandhi et al. 2010; Grobusch 2010). They include delayed treatment initiation, prior treatment with anti-tuberculosis drugs, use of fewer second-line drugs in a regimen, previously treatment with second-line drugs, prior exposure to fluoroquinolones, resistance to fluoroquinolones or to capreomycin, low BMI, HIV-seropositivity and other immunosuppressive conditions. Treatment outcome of MDR-TB patients from resource-limited settings reported cure rates of $60-75 \%$ (O’Donnell et al. 2009). The proportion of MDR-TB patients who were successfully treated ranged from $77 \%$ among new cases to $69 \%$ among previous treated patients. A recent report has confirmed that XDR-TB had higher probability of death, longer hospitalisation, longer treatment duration and delayed microbiological conversion when compared to MDR-TB at TB reference centres in Italy and Germany (Migliori et al. 2007). Earlier observations from the Tugela Ferry outbreak in the Republic of South Africa that XDR-TB is untreatable have not been confirmed in other areas of the world where HIV was not a big problem (Gandhi et al. 2010). A recent systematic review on MDR-TB and XDR-TB, including studies from North and South America, Europe and Korea (Sotgiu et al. 2009), has shown that XDR-TB can be successfully treated in more than $50 \%$ of patients. However, treatment duration is significantly longer, and outcomes are in general poorer than for non-XDR-TB patients.

Our latest data from South Africa demonstrated in a large cohort of XDR-TB cases $(n=220,43 \%$ being HIV-seropositive) that the overall mortality was $36 \%$, was similar in HIV-positive and HIV-negative individuals, but significantly lower in HIV-positive patients not treated with antiretroviral therapy compared to those who were (Dheda et al. 2010). Thus, overall treatment-related outcomes of XDR-TB were generally poor. This may be related to several factors operating in resource-poor settings, such as the high proportion of patients with prior MDR-TB, malnutrition, re-infection and strain dynamics, among other factors.

\section{Surgical treatment for MDR-TB and XDR-TB}

The role of surgery in the management of extensive pulmonary involvement in MDR-/XDR-TB remains controversial (Torun et al. 2007; Dravniece et al. 2009). Surgical resection of infected lung tissue has been reported to be a useful strategy in the treatment of MDR-TB and XDR-TB. While some studies reported that surgery was associated with a better outcome (Chiang et al. 2001; Somocurcio et al. 2007), others found no additional benefit of surgery in the treatment of MDR-TB (Torun et al. 2007). The contribution of surgical intervention may be useful when medical treatment fails to clear sputum mycobacteria (Chiang et al. 2001; Dravniece et al. 2009). Surgical intervention is usually indicated for extensive drug resistance with a high probability of failure of medical therapy alone (Iseman et al. 1990; Motus et al. 2006), but its timing is not yet well defined. Surgical intervention depends on the following two factors: (1) localised lung disease with good chances for complete or nearly complete resection and adequate expected post-operative lung function, and (2) susceptibility of M. tuberculosis isolate to anti-TB drugs to ensure post-surgery stump healing (Iseman et al. 1990). Effective anti-tuberculosis treatment offered for at least 3 months prior to the surgical intervention to reduce bacillary load is recommended (Iseman et al. 1990). Surgical interventions complementing individualised chemotherapy regimens guided by DST have led to treatment success rates in selected MDR-TB patients of $>90 \%$ in a number of studies, but success rates in patients with XDR-TB are lower. A study from Ekaterinburg (Russian Federation) evaluated the outcomes of 214 cultureconfirmed patients with cavitary pulmonary TB $(79.9 \%$ of them being MDR): 109 underwent artificial pneumothorax, and 105 were treated with chemotherapy only (Motus et al. 2006). Among new cases, those who had artificial pneumothorax had a higher proportion of sputum smear conversion than controls $(100 \%$ vs. $70.9 \% ; P<0.01)$. Similarly, among retreatment cases, the proportion of smear conversion was, respectively, $81 \%$ among cases $v$ s. $40 \%$ among controls $(P<0.01)$. The time to conversion was also faster in group than in control cases (Motus et al. 2006). Further studies are necessary to evaluate the role of artificial pneumothorax in treating MDR-/XDR-TB cases.

\section{Public health responses to drug-resistant TB epidemic}

\section{Infection control measures and recommendations}

WHO policy on TB infection control in health care settings and households has been clearly defined (WHO, 2009). This covers organisational activities (surveillance and assessment at all levels of the health system), administrative controls (triage, cough etiquette, reduction in unnecessary hospital stay, among others), environmental controls (natural ventilation, mechanical ventilation, ultra-violet irradiation and health facility renovation) and personal protection. Personal protection includes both the use of 
G. B. Migliori et al. Drug-resistant TB

respirators for health staff and masks for patients on one side and the 'Package of prevention and care for health care workers' on the other side [including HIV prevention, antiretroviral therapy (ART) and INH preventive therapy for HIV-positive health care workers].

Tuberculosis infection control measures in most health care facilities in the developing countries are virtually nonexistent because of poor investment by governments into their health systems and TB programmes (Kranzer et al. 2010). Implementation of comprehensive infection control measures is not usually feasible, but implementing simple triage systems to separate potentially infectious (coughing) patients, and providing masks to patients and/or staff could be easily carried out. Ideally, patients with drugresistant TB must be cared for in hospitals equipped with negative pressure isolation facilities, administrative hospital protocols to deal with such patients and appropriate environmental protective measures should be taken. Adequate ventilation (>12 air changes/h) obtained by opening windows and doors is the most important and easily implemented measure other than diagnosing and treating infectious cases early and effectively and separating suspected cases from other susceptible people such as children and HIV-infected individuals. Cough etiquette is also a cost-effective intervention that needs to be implemented at all levels. A recent modelling study on infection control outcomes estimated that half of anticipated XDRTB cases could be averted by applying a combination of available strategies in developing countries (WHO, 2009). Appropriate safety measures using negative pressure category 3 cabinets, masks and gloves should be implemented by laboratory staff when dealing with biological samples from patients suspected of having drug-resistant TB strains.

\section{Contact tracing of workmates and family members for drug-resistant TB}

By the time a patient is diagnosed with drug-resistant $\mathrm{TB}$, most of his/her contacts will have been infected. Both hospital and community-based health care workers are at risk of acquiring drug-resistant TB (Kranzer et al. 2010). Contact tracing is one of the most fundamental measures to detect active cases of TB, and these services must be aligned to all health services. Everyone diagnosed with drug-resistant TB must be isolated, and infection control procedures described earlier should be followed.

Importance of proper implementation of TB programme control measures to control drug-resistant $\mathrm{TB}$

There are only a limited number of treatment options for drug-resistant TB because the numbers of safe and effective
TB drugs are few. An urgent and comprehensive TB programme response to the drug-resistant $\mathrm{TB}$ epidemic is required to tackle a grave situation, which threatens to derail gains made in TB control. The extent of MDR-TB and XDR-TB in areas of high HIV prevalence needs to be defined. Laboratory capacity (trained staff and appropriate equipment) needs to be established to undertake DST to facilitate rapid diagnosis of drug resistance. The HIV/AIDS epidemic continues to generate large numbers of TB cases, and the close association between the two diseases poses major challenges to TB and HIV control programmes of all developing countries (Lalloo \& Pillay 2008; Abdool Karim et al. 2009). There is an urgent need for government national TB programmes to synergise their activities with HIV/AIDS programmes and ensure that control methods that are known to work are implemented. TB and HIV programmes must be aligned together and their capabilities strengthened to improve diagnostic capabilities, improved treatment rates and monitoring of drug-resistant TB.

Priorities for HIV-infected patients with TB are improved case detection rates, HIV screening procedures, initiating antiretroviral therapy and INH prophylactic therapy as appropriate, increasing treatment completion rates, accurately diagnosing drug-resistant cases by introducing the latest available technologies for rapid detection, effectively treating drug-resistant cases, implementing effective contact tracing and infection control measures. TB control programmes are dependent on the health systems they are part of and governments should wake up to the emergency that drug-resistant TB poses. They must ensure that greater emphasis is given to diagnosing and treating patients with $\mathrm{TB}$ and $\mathrm{TB} / \mathrm{HIV} / \mathrm{AIDS}$ based on existing principles of $\mathrm{TB}$ care.

\section{Global recommendations for prevention and control of drug-resistant TB}

In 2007, pressed by the emerging threat represented by MDR-TB and XDR-TB, WHO formulated a policy to address this epidemic at the global level (Shah et al. 2005; WHO and IUATLD, 2008; WHO, 2010). The major recommendations formulated by the expert panel were (i) to improve the 'quality' of TB control globally to prevent the selection of MDR-TB and XDR-TB mutants, and (ii) to further improve the organisation of early diagnosis and effective treatment of the existing cases. WHO developed a broader approach, known as the new Stop TB Strategy, built upon the existing well-known DOTS strategy as the element of continuity. The new strategy is composed of six elements: (1) pursue high-quality DOTS expansion and enhancement; (2) address TB/HIV, MDR-TB and other challenges; (3) promote health system strengthening; (4) 
engage all care providers; (5) empower people with TB and communities; and (6) enable, promote and fund research.

\section{Recent advances in rapid diagnostics and treatment for drug-resistant TB}

\section{New technological advances for rapid diagnostics for MDR-TB}

Current DST testing laboratory methods take a few weeks before the results are available; the need for rapid identification of drug-resistant TB is great. Newer molecular-based diagnostic technologies for rapid identification of drug-resistant genes from patient sputum samples, albeit very expensive presently, have been recently introduced (Pillay \& Sturm 2010; Wallis et al. 2010). These show promise for early diagnosis and rapid institution of effective chemotherapy. This would improve management and cure rates of patients with drug-resistant TB. This will also allow for national TB programmes to improve MDRTB surveillance, notification and instituting infection control. They include Gene Xpert (Cepheid), the line probe assays (e.g. Hain MDRplus assay and InnoLip assay) and the mycobacteriophage assays. For identification of drug resistance, bacteriophage amplification assays have shown high specificity and sensitivity in culture M. tuberculosis isolates, but evidence is lacking for their use on clinical specimens. Gene Xpert is a user-friendly 'closed' system nested real-time PCR assay that enables a rapid confirmation of TB and also simultaneously determines susceptibility to RMP. Formats to detect resistance to other drugs are under development. The line probe assays amplify a resistance-containing gene segment, e.g. the $r p o B$ gene 'hotspot', and the PCR products are hybridised to oligonucleotide probes on a nitrocellulose strip (line probe; this is an 'open' system) (Pai et al. 2006). A variation, the Hain MDRplus sl version, can rapidly detect resistance to several drugs. There are several studies currently evaluating these technologies at research field sites in high endemic TB areas. If found to be useful, the cost of these machines will drop to affordable levels as sales increase and donor funding is justified.

\section{Cheaper alternative technologies for diagnosis of drug-} resistant $\mathrm{TB}$

There are other accurate and cheap alternative methods described for use in resource-poor settings, and drug susceptibility results are often available within 2-3 weeks. These include microscopic observation DST (MODS), which relies on identifying the characteristic cording of M. tuberculosis in liquid medium using an inverted light microscope (Moore et al. 2006). The main drawback is that it is labour intensive. Thin-layer agar microscopically detects early growth of M. tuberculosis on a thin layer of agar; the platform can be simplified and standardised to detect resistance on colorimetric grounds. The nitrate reductase assays rely on a $M$. tuberculosis produced nitrite-induced colour change (Griess reaction) to detect growth in selective media (Martin et al. 2008). Novel approaches include the high resolution melt assay, which is able to identify clinical isolates harbouring mutations conferring RMP resistance by comparing DNA melt profiles of their RMP resistance determining region to the DNA melt profiles of a wild type standard (Hoek et al. 2008; Pietzka et al. 2009). The challenge is now to determine how these technologies will perform in high and low-burden MDR-TB settings, what impact they may have on outcomes, and how best to combine them with existing low cost diagnostic tools in high-burden settings. Several studies are ongoing at present evaluating how the Hain MDRplus assay performs when included as early as possible in diagnostic algorithms in high TB incidence, low income countries.

\section{Newer anti-TB drugs pipeline and drug regimens}

The Global Alliance for TB drug development has a number of drugs in the pipeline. These will soon be evaluated in good clinical practices (GCP) randomised controlled clinical trials for their to shorten the duration of anti-TB chemotherapy, to test their effectiveness for use in patients with drug-resistant TB (Lienhardt et al. 2010; Ma et al. 2010). The diarylquinoline Tibotec Medical Compound (TMC)207, which has a unique mode of action inhibiting mycobacterial ATP synthase, showed delayed bactericidal activity in early bactericidal activity studies in new patients with TB, with no serious TMC207 related adverse effects. Other promising bactericidal and potentially sterilising compounds currently evaluated in phase 1 trials and Early Bactericidal Activity (EBA) studies are the two nitroimidazoles, PA-824 (nitroimidazo-oxazine) and OPC-67683 (dihydroimidazo-oxazole), sudoterb (pyrrole LL-3858 and an EMB derivative (diamine SQ109). The process from drug discovery, safety and pharmacokinetic studies to phase I, I, III studies takes nearly 20 years before a product comes into the market. A new initiative called 'Critical Path to TB Regimens' (CPTR) consisting of industrial, funder and NGOs partners aims to drastically reduce the amount of time needed to register novel drug regimens for treatment of all forms of TB (TB Alliance, 2010). It is important that clinicians and public health officers are regularly updated on all new developments in this area. 
G. B. Migliori et al. Drug-resistant TB

\section{Potential immunotherapies for drug-resistant TB}

The sub-optimal treatment outcomes currently achieved for XDR-TB and MDR-TB cases call for innovative new strategies to increase treatment effectiveness. Adjunct immunotherapy to drug treatment has been discussed for some time now. The aim of immunotherapy is to 'realign' or improve the immune response by either promoting protective (Th1) immunity or blocking harmful immune (Th2) responses. Currently available immunotherapeutic agents can be divided into three categories: immunoregulatory approaches, immunosuppressive therapy and supplement effector cytokines.

Immunoregulatory approaches are seeking to alter the nature of the immune response (Churchyard et al. 2009). They can be divided into three sub-groups, those for which (i) good manufacturing practices (GMP) manufacturing capacity exists (High dose IVIg, HE2000-16 $\alpha$-bromoepiandrosterone, multidose heat-killed Mycobacterium vaccae or Mycobacterium w, anti-IL-4), those for which (ii) GMP manufacturing capacity can be established [DNA vaccine (HSP65)] and (iii) the others (Dzherelo, SCV-07 SciCLone, RUTI).

Immunosuppressive therapy seeks to increase access of antibacterial drugs, or their susceptibility to them. They include thalidomide (lowering TNF levels), etanercept (blocking TNF) and prednisolone (having a broad anti-inflammatory effect). Supplement effector cytokines are aimed at enhancing the anti-microbicidal activity of the existing drugs. They include recombinant human (rh) IFN-gamma and rh-IFN-gamma, rh-IL-2, rh-granulocytemacrophage colony-stimulating factor (GM-CSF) and IL12. Studies are being planned to evaluate the use of adjunct immunotherapy (e.g. multiple doses of either M. vaccae, M. $w$ or IVIG) for the treatment of MDR-TB and XDR-TB patients. They will be conducted in parallel involving study cohorts in South Africa and Eastern Europe. As this is a novel area, it will be several more years before efficacy data are available and included (if proven to be effective) in programmatic management of MDR-TB and XDR-TB cases in resource-limited settings.

\section{Global efforts to tackle MDR-TB and XDR-TB}

Further key steps in the fight against MDR-TB and XDRTB are documented by the Global MDR-TB and XDR-TB Response Plan and the outcomes of the governmental conference organised in China in April 2009 to develop the Beijing Call for Action (World Health Organization, 2007). The document committed the 27 high-MDR-TB burden countries to undertake a specific set of actions for MDR-TB and XDR-TB containment and prevention.

Recommendations for increased investment by national TB Programmes in HIV/TB endemic areas are as follows:

(1) Preventing XDR-TB through basic strengthening and alignment of TB and HIV programmes by increasing case detection and effective treatment of drug sensitive TB. The new Stop TB strategy and the Global Plan to Stop TB are the key reference documents to guide these priority interventions.

(2) Improving management of individuals suspected to be affected by MDR-TB and XDR-TB through accelerated access to laboratory facilities with rapid DST test for RMP and INH resistance, DST for MDR-TB cases and improved detection of cases suspecting of harbouring MDR strains both in high and in low HIV prevalence settings.

(3) Strengthening management of MDR and XDR-TB and treatment design in both HIV-negative and positive individuals, through adequate use of secondline drugs and patient-centred approaches to ensure support and supervision.

(4) Standardising the definition of MDR-TB and XDRTB.

(5) Increasing contact tracing and screening.

(6) Health care worker infection control and protection mainly (but not exclusively) in high HIV prevalence settings.

(7) Implementing immediate MDR-TB and XDR-TB surveillance activities.

(8) Initiating advocacy, communication and social mobilisation activities to inform and raise awareness about TB and drug-resistant TB.

Within the framework of these recommendations, USAID in collaboration with WHO and other partners has developed a tool (known as 'MDR/XDR-TB Assessment and Monitoring Tool') to be used for (i) preparing national or sub-national plans for MDR/XDR-TB prevention and control; (ii) providing baseline information and monitoring progress; (iii) providing data and analysis to prepare Green Light Committee (GLC) and Global Fund to Fight AIDS, Tuberculosis and Malaria (GFATM) applications; (iv) providing information to guide requests for external technical assistance; and $(\mathrm{v})$ providing information to guide donor investment in MDR-/XDR-TB interventions. This suggests that using existing interventions, public health systems and TB and HIV programmes must be strengthened significantly, and political and funder commitment is essential to curb the spread of drug-resistant TB. 


\section{Acknowledgements}

KD and this work was supported by a SA Research Chair Initiative award, a MRC Career Development Fellowship, the EU(FP7-TBsusgent) and the EDCTP. AZ, PM, MB, and $\mathrm{MH}$ receive support from the EU-FP7, EDCTP, Global Alliance for TB Drug development, EuropeAID-ADAT, and AZ from the UK MRC and the UK NIHR UCLHCBRC. Adam Zumla kindly designed Figure 1 and provided administrative assistance.

\section{References}

Abdool Karim SS, Churchyard GJ, Abdool Karim Q \& Lawn SD (2009) HIV infection and tuberculosis in South Africa: an urgent need to escalate the public health response. Lancet 374(9693), 921-933.

Amor Y, Nemser B, Singh A, Sankin A \& Schluger N (2008) Underreported threat of multidrug-resistant tuberculosis in Africa. Emerging Infectious Diseases 14, 1345-1352.

Balabanova Y, Ruddy M, Hubb J et al. (2005) Multidrug-resistant tuberculosis in Russia: clinical characteristics, analysis of second-line drug resistance and development of standardized therapy. European Journal of Clinical Microbiology and Infectious Diseases 24, 136-139.

Caminero JA (2008) Likelihood of generating MDR-TB and XDRTB under adequate National Tuberculosis Programme implementation. The International Journal of Tuberculosis and Lung Disease 12, 869-877.

Centers for Disease Control and Prevention (2006) Emergence of Mycobacterium tuberculosis with extensive resistance to secondline drugs-worldwide. Morbidity and Mortality Weekly Report 55, 301-305.

Chan ED, Laurel V, Strand MJ et al. (2004) Treatment and outcome analysis of 205 patients with multidrug-resistant tuberculosis. American Journal of Respiratory and Critical Care Medicine 169, 1103-1109.

Chiang C-Y, Yu M-C, Bai K-J, Lin T-P \& Lee Y-C (2001) Pulmonary resection in the treatment of patients with pulmonary multidrug-resistant tuberculosis in Taiwan. The International Journal of Tuberculosis and Lung Disease 5, 272-277.

Chiang C-Y, Enarson DA, Yu M-C et al. (2006) Outcome of pulmonary multidrug-resistant tuberculosis: a 6-yr follow-up study. European Respiratory Journal 28, 980-985.

Chiang C-Y, Caminero JA \& Enarson DA (2009) Reporting on multidrug-resistant tuberculosis: a proposed definition for the treatment outcome 'failed". The International Journal of Tuberculosis and Lung Disease 13, 548-550.

Chirenda J, Menzies H, Moalosi G et al. (2009) The trend of resistance to anti-tuberculosis drugs in Botswana: results from the 4th national anti-tuberculosis drug resistance survey. 40th Union World Conference on Lung Health. Int J TB Lung Dis, Cancun, Mexico.

Churchyard G, Kaplan G, Fallows D, Wallis RS, Onyebujoh P \& Rook GA (2009) Advances in immunotherapy for Tuberculosis treatment. Clinics in Chest Medicine. Tuberculosis volume, Eds A. Zumla and S. Schaaf. 30(4), 769-796.

Dheda K, Shean K, Zumla A et al. (2010) Early treatment outcomes of extensively drug-resistant tuberculosis in South Africa are poor regardless of HIV status. The Lancet 375, 1798-1807.

Dravniece G, Cain KP, Holtz TH, Riekstina V, Leimane V \& Zaleskis R (2009) Adjunctive resectional lung surgery for extensively drug-resistant tuberculosis. European Respiratory Journal 34, 180-183.

Dubrovina I, Miskinis K, Lyepshina S et al. (2008) Drug-resistant tuberculosis and HIV in Ukraine: a threatening convergence of two epidemics? The International Journal of Tuberculosis and Lung Disease 12(7),756-762.

Elliott AM, Halwiindi B, Hayes RJ et al. (1993) The impact of human immunodeficiency virus on presentation and diagnosis of tuberculosis in a cohort study in Zambia. Journal of Tropical Medicine and Hygiene 96, 1-11.

Espinal MA (2003) Time to abandon the standard retreatment regimen with first-line drugs for failures of standard treatment. The International Journal of Tuberculosis and Lung Disease 7, 607-608.

Espinal MA, Kim SJ, Suarez PG et al. (2000) Standard shortcourse chemotherapy for drug-resistant tuberculosis. Treatment outcomes in 6 countries. JAMA 283, 2537-2545.

Espinal MA, Laszlo A, Simonsen L et al. (2001) Global trends in resistance to antituberculosis drugs. World Health Organization-International Union against Tuberculosis and Lung Disease Working Group on Anti-Tuberculosis Drug Resistance Surveillance. New England Journal of Medicine 344(17), 1294-1303.

Flament-Saillour M, Robert J, Jarlier V \& Grosset J (1999) Outcome of multi-drug-resistant tuberculosis in France. A nationwide case-control study. American Journal of Respiratory and Critical Care Medicine 160, 587-593.

Gandhi NR, Moll A, Sturm AW et al. (2006) Extensively drugresistant tuberculosis as a cause of death in patients co-infected with tuberculosis in a rural area of South Africa. The Lancet 368, 1575-1580.

Gandhi NR, Shah NS, Andrews JR et al. (2010a) HIV coinfection in multidrug- and extensively drug-resistant tuberculosis results in high early mortality. Tugela Ferry Care and Research (TF CARES) Collaboration. American Journal of Respiratory and Critical Care Medicine 181(1), 80-86.

Gandhi N, Nunn P, Dheda K et al. (2010b) Multidrug-resistant and extensively drug-resistant tuberculosis: a threat to global control of tuberculosis. The Lancet 375(9728), 1830-1843.

Goble M, Iseman MD, Madsen LA, Waite D, Ackerson L \& Horsburgh CR Jr (1993) Treatment of 171 patients with pulmonary tuberculosis resistant to isoniazid and rifampin. New England Journal of Medicine 328, 527-532.

Grobusch MP (2010) Drug-resistant and extensively drug-resistant tuberculosis in southern Africa. Current Opinion in Pulmonary Medicine 16(3), 180-185.

Habeenzu C, Mitarai S, Lubasi D et al. (2007) Tuberculosis and multidrug resistance in Zambian prisons, 2000-2001. The International Journal of Tuberculosis and Lung Disease 11, 1216-1220. 
G. B. Migliori et al. Drug-resistant TB

Hoek KG, Gey van Pittius NC, Moolman-Smook H et al. (2008) Fluorometric assay for testing rifampin susceptibility of $\mathrm{Myco-}$ bacterium tuberculosis complex. Journal of Clinical Microbiology 46, 1369-1373.

Holtz TH \& Cegielski JP (2007) Origin of the term XDR-TB. European Respiratory Journal 30, 396.

Holtz TH, Riekstina V, Zarovska E et al. (2005) XDR-TB: extreme drug-resistance and treatment outcome under DOTS-Plus, Latvia, 2000-2002. The International Journal of Tuberculosis and Lung Disease 9(Suppl. 1), S258.

Iseman MD, Madsen L, Goble M \& Pomerantz M (1990) Surgical intervention in the treatment of pulmonary disease caused by drug-resistant Mycobacterium tuberculosis. The American Review of Respiratory Disease 141, 623-625.

Johnson R, Streicher E, Louw GE et al. (2009) Drug resistance in Mycobacterium tuberculosis. Current Issues in Molecular Biology 8, 97-112.

Kehinde AO, Obaseki FA, Ishola OC \& Ibrahim KD (2007) Multidrug resistance to Mycobacterium tuberculosis in a tertiary hospital. Journal of the National Medical Association 99, 11851189.

Kim HJ, Hong YP, Kim SJ, Lew WJ \& Lee EG (2001) Ambulatory treatment of multidrug-resistant pulmonary tuberculosis patients at a chest clinic. The International Journal of Tuberculosis and Lung Disease 5, 1129-1136.

Kingkaew N, Sangtong B, Amnuaiphon W et al. (2009) HIVassociated extrapulmonary tuberculosis in Thailand: epidemiology and risk factors for death. International Journal of Infectious Diseases 13(6), 722-729.

Korenromp EL, Scano F, Williams BG et al. (2003) Effects of human immunodeficiency virus infection on recurrence of tuberculosis after rifampin-based treatment: an analytical review. Clinical Infectious Diseases 37, 101-112.

Kranzer K, Bekker LG, Van Schaik N et al. (2010) Community health care workers in South Africa are at increased risk for tuberculosis. South African Medical Journal 100(4), 224-226. No abstract available.

Lalloo UG \& Pillay S (2008) Managing tuberculosis and HIV in sub-Sahara Africa. Current HIV/AIDS Reports 5(3), 132-139. Review.

Laserson KF, Thorpe LE, Leimane V et al. (2005) Speaking the same language: treatment outcome definitions for multidrugresistant tuberculosis. The International Journal of Tuberculosis and Lung Disease 9, 640-645.

Lienhardt C, Vernon A \& Raviglione MC (2010) New drugs and new regimens for the treatment of tuberculosis: review of the drug development pipeline and implications for national programmes. Current Opinion in Pulmonary Medicine 16(3), 186-193.

Ma Z, Lienhardt C, McIlleron H, Nunn AJ \& Wang X (2010) Global tuberculosis drug development pipeline: the need and the reality. The Lancet [Epub ahead of print].

Martin A, Panaiotov S, Portaels F, Hoffner S, Palomino JC \& Angeby K (2008) The nitrate reductase assay for the rapid detection of isoniazid and rifampicin resistance in Mycobacterium tuberculosis: a systematic review and meta-analysis. Journal of Antimicrobial Chemotherapy 62, 56-64.
Matteelli A, Migliori GB, Cirillo D, Centis R, Girard E \& Raviglion M (2007) Multidrug-resistant and extensively drugresistant Mycobacterium tuberculosis: epidemiology and control. Expert Review of Anti-infective Therapy 5(5), 857-871.

Meskel DW, Abate G, Lakew M, Goshu S \& Aseffa A (2008) Antituberculosis drug resistance among retreatment patients seen at St Peter Tuberculosis Specialized Hospital. Ethiopian Medical Journal 46, 219-225.

Migliori GB, Espinal M, Danilova ID, Punga VV, Grzemska M \& Raviglione MC (2002) Frequency of recurrence among MDRTB cases 'successfully' treated with standardised short-course chemotherapy. The International Journal of Tuberculosis and Lung Disease 6, 858-864.

Migliori GB, Besozzi G, Girardi E et al. (2007a) Clinical and operational value of the extensively drug-resistant tuberculosis definition. European Respiratory Journal 30(4), 623-626.

Migliori GB, Ortmann J, Girardi E et al. (2007b) Extensively drug-resistant tuberculosis, Italy and Germany. Emerging Infectious Diseases 13(5), 780-782.

Migliori GB, Richardson MD, Sotgiu G \& Lange C (2009) Multidrug-resistant and extensively drug-resistant tuberculosis in the West, Europe and United States: epidemiology, surveillance, and control. Clinics in Chest Medicine 30, 637-665.

Migliori GB, Centis R, Lange C, Richardson MD \& Sotgiu G (2010) Emerging epidemic of drug-resistant tuberculosis in Europe, Russia, China, South America and Asia: current status and global perspectives. Current Opinion in Pulmonary Medicine 16(3), 171-179.

Moore DA, Evans CA, Gilman RH et al. (2006) Microscopicobservation drug-susceptibility assay for the diagnosis of TB. New England Journal of Medicine 355, 1539-1550.

Motus IY, Skorniakov SN, Sokolov VA et al. (2006) Reviving an old idea: can artificial pneumothorax play a role in the modern management of tuberculosis? The International Journal of Tuberculosis and Lung Disease 10, 571-577.

O’Donnell MR, Padayatchi N, Master I, Osburn G \& Horsburgh CR (2009) Improved early results for patients with extensively drug-resistant tuberculosis and HIV in South Africa. The International Journal of Tuberculosis and Lung Disease 13(7), 855-861.

Pablos-Méndez A, Raviglione MC, Laszlo A et al. (1998) Global surveillance for antituberculosis-drug resistance, 1994-1997. The New England Journal of Medicine 338(23), 1641-1649.

Pai M, Kalantri S \& Dheda K (2006) New tools and emerging technologies for the diagnosis of tuberculosis: part II. Active tuberculosis and drug resistance. Expert Review of Molecular Diagnostics 6, 423-432.

Park SK, Kim CT \& Song SD (1998) Outcome of chemotherapy in 107 patients with pulmonary tuberculosis resistant to isoniazid and rifampicin. The International Journal of Tuberculosis and Lung Disease 2, 877-884.

Pietzka AT, Indra A, Stoger A et al. (2009) Rapid identification of multidrug-resistant Mycobacterium tuberculosis isolates by rpoB gene scanning using high-resolution melting curve PCR analysis. Journal of Antimicrobial Chemotherapy 63, 11211127. 
G. B. Migliori et al. Drug-resistant TB

Pillay M \& Sturm AW (2010) Nosocomial transmission of the F15/LAM4/KZN genotype of Mycobacterium tuberculosis in patients on tuberculosis treatment. The International Journal of Tuberculosis and Lung Disease 14(2), 223-230.

Quy HTW, Lan NTN, Borgdorff MW et al. (2003) Drug resistance among failure and relapse cases of tuberculosis: is the standard re-treatment regimen adequate? The International Journal of Tuberculosis and Lung Disease 7, 631-636.

Saravia JC, Appleton SC, Rich ML, Sarria M, Bayona J \& Becerra MC (2005) Retreatment strategies when first-line tuberculosis therapy fails. The International Journal of Tuberculosis and Lung Disease 9, 421-429.

Schaaf S \& Dheda K (2009) Multidrug and extensively drugresistant tuberculosis in Africa and South America: epidemiology, diagnosis and management in adults and children. Clinics in Chest Medicine Eds A. Zumla and S. Schaaf. Tuberculosis volume 300(4), 667-683.

Schaaf S \& Zumla A Eds (2009) Tuberculosis - A Comprehensive Clinical Reference, 107 Chapters. Publishers Elsevier Saunders, UK.

Shah NS, Wright A, Drobniewski F et al. (2005) Extreme drug resistance in tuberculosis ("XDR-TB"): global survey of supranational reference laboratories for Mycobacterium tuberculosis with resistance to second-line drugs. The International Journal of Tuberculosis and Lung Disease 9(Suppl 1), S77.

Somocurcio JG, Sotomayor A, Shin S et al. (2007) Surgery for patients with drug-resistant tuberculosis: report of 121 cases receiving community-based treatment in Lima, Peru. Thorax 62, 416-421.

Sonnenberg P, Murray J, Glynn JR et al. (2001) HIV-1 and recurrence, relapse, and reinfection of tuberculosis after cure: a cohort study in South African mineworkers. Lancet 358, 1687-1693.

Sotgiu G, Ferrara G, Matteelli A et al. (2009) Epidemiology and clinical management of XDR-TB: a systematic review by TBNET. European Respiratory Journal 33(4), 871-881.

TB Alliance (2010) Global alliance website http://www.tballinace. org/cptr/ (accessed 26 May 2010).
Torun T, Tahaoglu K, Ozmen I et al. (2007) The role of surgery and fluoroquinolones in the treatment of multidrug-reistant tuberculosis. The International Journal of Tuberculosis and Lung Disease 11, 979-985.

Umubyeyi AN, Vandebriel G, Gasana M et al. (2007) Results of a national survey on drug resistance among pulmonary tuberculosis patients in Rwanda. The International Journal of Tuberculosis and Lung Disease 11, 189-194.

Wallis RS, Pai M, Menzies D et al. (2010) Biomarkers and diagnostics for tuberculosis: progress, current needs and translation into practice. The Lancet 375, 1920-1937.

WHO (2009) WHO Policy on TB Infection Control in Healthcare Settings, Congregate Settings and Households. World Health Organization, Geneva.

WHO (2010) WHO Report 2010: Multidrug and Extensively Drug-resistant TB (MDR-/XDR-TB). 2010 Global Report on Surveillance and Response. WHO, Geneva. $\mathrm{WHO} / \mathrm{HTM} / \mathrm{TB} / 2010.3$.

WHO and IUATLD (2008) Anti-tuberculosis Drug Resistance in the World: Fourth Global Report. World Health Organization, Geneva.

World Health Organization (2007) The global MDR-TB \& XDRTB response plan 2007-2008. World Health Organization Document 2007; WHO/HTM/STB/2007.2007.387:1-s51.

Wright A, Zignol M, Van Deun A et al.; for the Global Project on Anti-Tuberculosis Drug Resistance Surveillance (2009a) Epidemiology of antituberculosis drug resistance 2002-07: an updated analysis of the Global Project on Anti-Tuberculosis Drug Resistance Surveillance. Lancet 373(9678), 18611873.

Wright A, Zignol M, Van Deun A et al. (2009b) Epidemiology of antituberculosis drug resistance 2002-07: an updated analysis of the Global Project on Anti-Tuberculosis Drug Resistance Surveillance. Lancet 373, 1861-1873.

Zumla A \& Schaaf S, Eds (2009) Clinics in Chest Medicine. Tuberculosis Volume, December 2009. Publishers Elsevier Saunders, Philadelphia, USA, p. 13.

Corresponding Author Alimuddin Zumla, Centre for Infectious Diseases and International Health, Department of Infection, University College London Medical School, London W1T 4JF, UK. E-mail: a.zumla@ucl.ac.uk 